\title{
NOTES
}

\section{Kinetic Study on the Formation of Polyelectrolyte Complexes. Poly(L-lysine hydrobromide) and Poly(L-glutamate sodium salt)}

\author{
Hiroko SATo ${ }^{\dagger}$ and Hiroyuki SeKI* \\ Department of Polymer Chemistry, Kyoto University, Kyoto 606-01, Japan and \\ Hahn-Meitner-Institute für Kernforschung Berlin GmbH, \\ Bereich Strahlenchemie, 1 Berlin 39, Germany
}

(Received November 9, 1992)

KEY WORDS Polyelectrolyte Complexes / Kinetic Study / Half Reaction Time / Poly(L-lysine $\mathrm{HBr})$ / Poly(L-glutamate Na)

The reaction of polycations and polyanions in aqueous solutions forms polyelectrolyte complexes (PEC), which results in solid-liquid phase separation, liquid-liquid phase separation, i.e., complex coacervation, or no phase separation, depending on the molecular properties, concentrations of polyelectrolytes, ionic strength and $\mathrm{pH}$ of media, charge density, molecular weight, combination of polycations and polyanions and so on. The reaction of PEC is not only applied in various flocculants and biotechnology, but found frequently in biological systems. However, less kinetic studies have been reported. Kinetics on PEC formation was studied by the conductance stopped-flow method $^{1}$ and by using luminescence-labeled protein. ${ }^{2}$ Two stages of relaxation time, obtained by the chemical relaxation method, ${ }^{3}$ were observed for the formation of PECs, soluble complexes, of polybrene and poly(acrylate- $\mathrm{Na}$ ) or poly(ethylenimine) and poly(ethylene sulfate-Na). One rapid relaxation time was considered to be attributed to electrostatic interaction and dehydration. The other slow relaxation time was regarded to result from the reorientation of the PEC formed.

In this work, a kinetic study was carried out for PECs which were capable of forming precipitates. $^{4,5}$ In such solid-liquid phase separation, the PEC formation may be kinetically observed as the change of turbidity or light scattering in solutions.

\section{EXPERIMENTAL}

\section{Materials}

Poly(L-lysine hydrobromide) (PLL-HBr, average molecular weight $M_{\mathrm{r}}, 70000$, Sigma Chemical Co.) was used as polycation, while sodium poly(L-glutamate) (PLG-Na, $M_{\mathrm{r}}$, 70000 , Sigma) was used as polyanion. Concentrations (equiv $1^{-1}$ ) of PLL-HBr or PLG$\mathrm{Na}$ were designated with respect to repeating units of ionizable groups on polyelectrolytes in solutions.

Potassium poly(vinyl sulfate) (PVS-K, Sigma) and polyvinyl pyrrolidon (PVP, $M_{r}$, 360000, EGA Chemie) were compared in the light scattering measurement. This PVS-K had one sulfate group per 5.8 repeating units of vinyl alcohol, which was estimated by conductometry after replacing the acidic form of the sulfate group with the anion and cation exchange resins (Dow Chemical, Midland, MI). The specific viscosity of $0.146 \mathrm{~g} \mathrm{dl}^{-1}$ PVS-H in water was 0.826 , which was mea-

$\dagger$ To whom correspondence should be addressed.

* Left from The Institute of Physical and Chemical Research, Wako, Saitama 351-01, Japan. 
sured with an Ostwald type viscometer at $27^{\circ} \mathrm{C}$. Distilled and deionized water, used in this work, was passed through the filter paper of pore size $0.01 \mathrm{~mm}$ (Sartrius Membranefilter $\mathrm{GmbH}$ ) and degassed before the light scattering measurement. Polymer solutions were passed through the filter paper of pore size $0.45 \mathrm{~mm}$ (Sartrius Membranefilter $\mathrm{GmbH}$ ). The PEC formation of PLL-HBr and PLG-Na were carried out in $0.05 \mathrm{M}$ phosphate buffer at $\mathrm{pH} 7.0$ containing $0.2 \mathrm{M} \mathrm{NaCl}$. With respect to PLL-HBr and PVS-K, the PEC formation was measured in aqueous solutions without adding microsalts at room temperature.

\section{Apparatus}

A polycation solution and a polyanion solution were mixed with a stopped flow apparatus (Durrum Instrument, Palo Alto, CA) for the kinetic study. The intensity of light scattering (LSI) of PEC formed in aqueous solutions was detected at right angles against the incidental beam of argon-ion laser (wave length, $514 \mathrm{~nm}$ and output $0.3 \mathrm{~W}$, Spectra Physics Model 165-00). The signal of photomultiplier $(800 \mathrm{~V}$, Hahn-Meitner-Inst, HAMI, 9781B) was transmitted to a 549 storage oscilloscope Type 203 (Tektronix, Heerenveen, The Netherland) or a recorder (HAMI). The dead time of the stopped flow apparatus was ranged within the delay time $13 \mathrm{~ms}$, fixed on an oscilloscope. An optical cuvette was made of quartz, and one shot extrusion volume was $0.15 \mathrm{ml}$.

\section{RESULTS AND DISCUSSION}

The PEC formation of PLL-HBr and PLG-Na was observed as the change of light scattering immediately after mixing both polyelectrolyte solutions (Figure 1). The initial rapid increase and subsequent gradual decrease through the maximum LSI ( LSI $_{\text {max }}$ ) were generally observed in the PEC formation, and the initial changes from mixing to $\mathrm{LSI}_{\max }$ were analyzed. In addition, LSI values of water

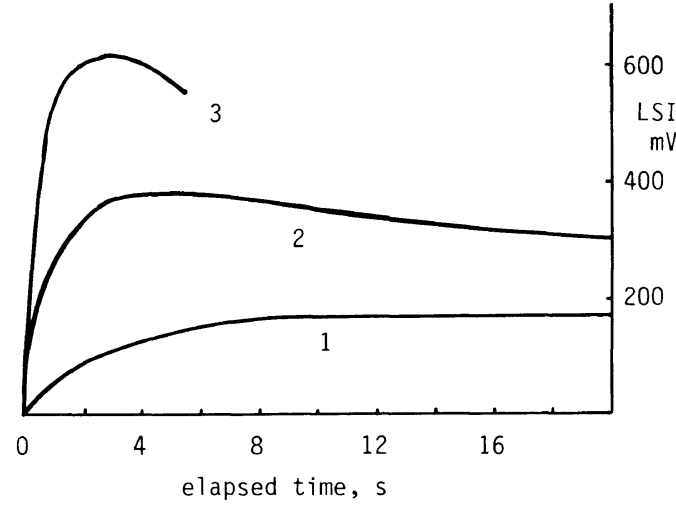

Figure 1. Change of light scattering intensity (LSI), caused by mixing PLL-HBr and PLG-Na solutions equivalently. Concentrations of total polyelectrolytes were $0.2,0.5$, and 1.0 mequiv $1^{-1}$ in curves 1,2 , and 3 , respectively.

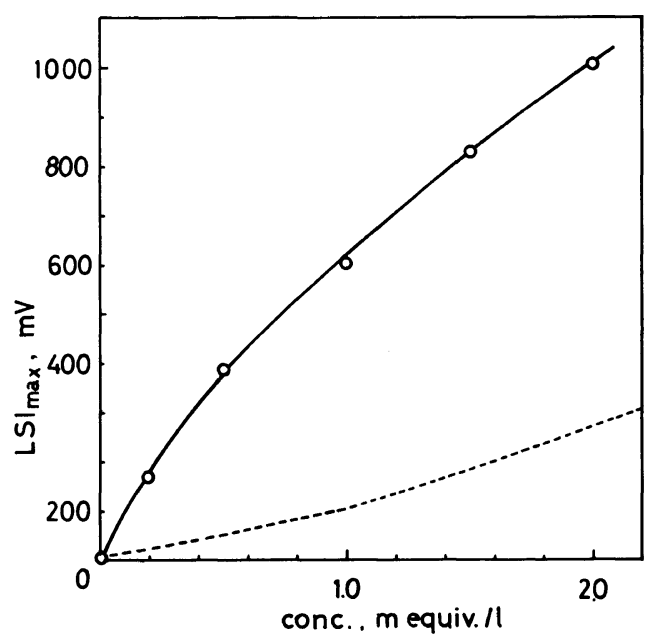

Figure 2. Dependence of $\mathrm{LSI}_{\max }$ values on concentrations of total polyelectrolytes (mequiv ${ }^{-1}$ ) in PEC solutions. $R_{\mathrm{pc}}=0.5$ for PLL-HBr and PLG-Na. Broken line corresponds to PEC of PLL-HBr and PVS-K at $R_{\mathrm{pc}}=0.568$.

used, $\quad 0.1 \%$ PLL-HBr aqueous solution, $0.16 \%$ PVS-K aqueous solution, and above $0.4 \%$ to $0.8 \%$ PVP aqueous solutions were respectively from 6 to $5 \mathrm{mV}, 6.7 \mathrm{mV}, 6.8 \mathrm{mV}$, and $9.6 \mathrm{mV}$. Therefore, the contribution of soluble complexes to LSI seems negligibly small in the system of PLL-HBr and PLG-Na, taking into consideration the value of $\mathrm{LSI}_{\max }$, $606 \mathrm{mV}$ at concentration of $1 \mathrm{~m}$ equiv $1^{-1}$ 
$(=0.0180 \%)$.

The mixing ratio $R_{\mathrm{pc}}$ is represented by

$$
R_{\mathrm{pc}}=N_{\mathrm{pc}} /\left(N_{\mathrm{pc}}+N_{\mathrm{pa}}\right)
$$

where $N_{\mathrm{pc}}$ and $N_{\mathrm{pa}}$ imply the total number (or concentrations expressed with equiv $1^{-1}$ ) of ionizable groups of polycation and polyanion molecules in solutions. Figure 2 shows the relationship between $\mathrm{LSI}_{\max }$ and total concentrations of polyelectrolytes in the equivalent mixing $\left(R_{\mathrm{pc}}=0.5\right)$ of polycations and polyanions. The amount of PEC formation for PLL(H)-PLG increased, bringing about a convex curve with increasing concentrations, while that of the PEC formation for PLL(H)PVS brought about a slightly concave curve. Although the weight per equivalence of PVS is larger by 2.9 times than PLG, the PEC precipitates of PLL(H)-PVS caused remarkably less LSI than those of PLL(H)-PLG, which should reflect the difference between both polyanions, e.g., compatibility and hydrophilicity against the aqueous solvent.

The reaction rate of $\mathrm{PEC}$ formation in the relative early stage was analyzed on the basis

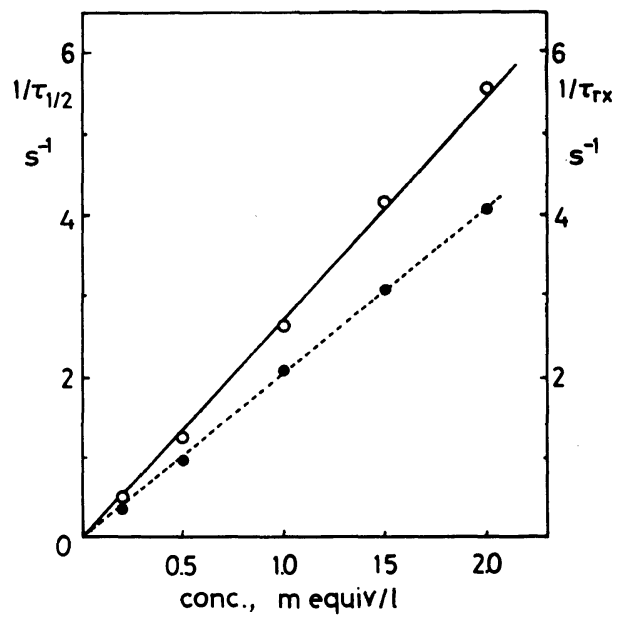

Figure 3. Dependence of the reciprocal of $t$ on concentrations of polyelectrolytes in equivalent mixing of PLL-HBr and PLG-Na. The solid line indicates the reciprocal of half reaction time $\left(\tau_{1 / 2}\right)^{-1}$ for the change of LSI. Broken line corresponds to the reciprocal of chemical relaxation time $\left(\tau_{\mathrm{rx}}\right)^{-1}$. of LSI-time (t) curves as shown in Figure 1. One index ${ }^{2,6}$ for the reaction rate was obtained from the reciprocal of the half reaction time, $\left(\tau_{1 / 2}\right)^{-1}$, i.e., the time, attained to half of the LSI $_{\max }$ value. As another index, chemical relaxation time, $\tau_{\mathrm{rx}}$, was determined according to the relaxation method, ${ }^{1,3}$ where the final LSI value for the analysis was obtained by extrapolating the initial and straight portion in the region over $\mathrm{LSI}_{\max }$ to $t=0$. Thus, two indeces for PEC formation are plotted on two straight lines against concentrations of polyelectrolytes, passing the origin (Figure 3 ). The relationship is expressed by

$$
\left(\tau_{\mathrm{rx}}\right)^{-1}=0.743\left(\tau_{1 / 2}\right)^{-1}
$$

Although the coefficient on eq 2 is calculated to be higher by $7.8 \%$ than $\ln 2$, the reciprocals of both indeces result in a good correlation.

Figure 4 shows the rate of PEC formation in various mixing ratios at 1 mequiv $1^{-1}$ for mixtures of PLL-K and PLG-Na. It is obvious that the rate of PEC formation is the highest

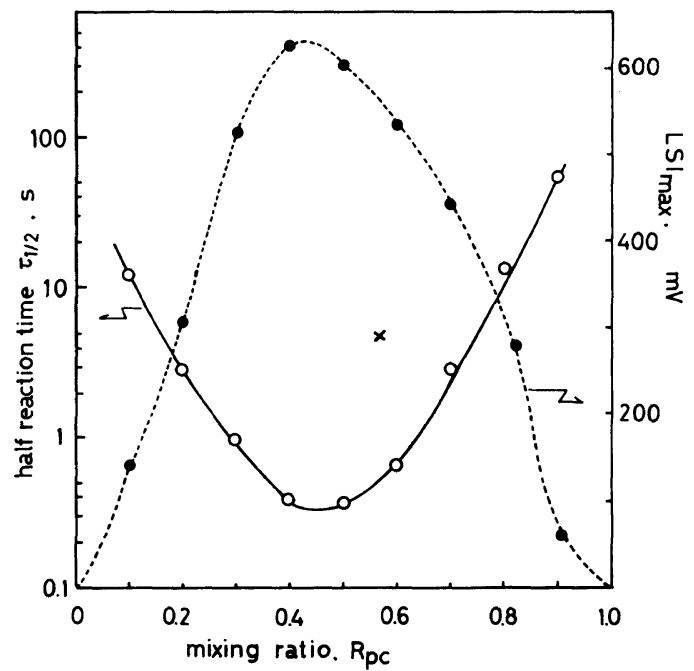

Figure 4. Relation between half reaction time $\left(\tau_{1 / 2}\right)$ of $\mathrm{PEC}$ formation and various mixing ratio $R_{\mathrm{pc}}$ of PLL-HBr and PLG-Na. The total concentration of PLL-HBr and PLG-Na was 1 mequiv $1^{-1}$. Cross mark corresponds to the rate of PEC formation for PLL-HBr and PVS-K $(0.127 \%$;

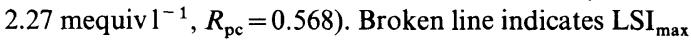
on the right ordinate against various mixing ratio of PLL-HBr and PLG-Na. 
around the region of equivalent mixing. LSI $_{\max }$ values were also remarkably high around the region where PEC was observed to form most rapidly as well as with the highest yield from polyelectrolyte solutions than in unequivalent mixing. In addition, the rate of PEC formation for PLL(H)-PVS was somewhat slower than that of PEC formation for PLL(H)-PLG.

The PEC formation of PLL-HBr and PLG$\mathrm{Na}$ however, shifts slightly from the stoichiometric reaction $\left(R_{\mathrm{pc}}=0.5\right)$ to 0.45 . This shift may correspond to the conductometric observation that a break was seen at 0.9 $\left(R_{\mathrm{pc}}=0.47\right)$ in a mixing ratio of PLL-HBr vs. PLG (Figure 1 in ref 5). In aqueous solution at $\mathrm{pH} 7.0$ without adding microsalts, the degree of dissociation for PLL and PLG was estimated to be 0.95 and 0.93 , respectively. ${ }^{4}$ The presence of $0.2 \mathrm{M} \mathrm{NaCl}$ may accelerate the dissociation of unionized groups on PLL and PLG. $^{7}$ Moreover, the induction ${ }^{7}$ of dissociation between anionic and cationic ionizable groups on polyelectrolytes should result in the PEC reaction. Hence, the degree of dissociation of PLL-HBr and PLG-Na may be regarded as almost 1 in this work. Therefore, the shift from stoichiometry in the PEC formation appears not to be due to the slight difference in concentrations between positive and negative net charges on polyelectrolytes. Another possibility of the shift from stoichiometry may be attributed to the conformational change of peptide chains in the PEC formation. From PLL-HBr or PLG-Na in random coil structure at $\mathrm{pH} 7$, the formation of $\beta$-structure in PLL(H)-PLG complex was observed from circular dichroism measurement. ${ }^{5}$ Such conformational changes are considered to make incomplete pairing between polycations and polyanions.

Two kinds of chemical relaxation times were reported in the formation of soluble complexes, ${ }^{1} \tau_{\mathrm{rx} 1}$ and $\tau_{\mathrm{rx} 2}$; values for $\tau_{\mathrm{rx} 1}$, less than $50 \mathrm{~ms}$, were observed for the initial and rapid increase in conductance in the presence of $5 \mathrm{mM} \mathrm{NaBr}$. On the other hand, $\tau_{\mathrm{rx} 2}$ values from 0.4 to $10 \mathrm{~s}$, depending on the degree of dissociation and concentrations of polyelectrolytes, were obtained in the magnitude of reaction time similar to our PEC formation. Hence, the formation of PEC precipitates should follow the pairing between polycations and polyanions, and our $\tau_{\mathrm{rx}}$ values, measured by light scattering, are regarded to involve both electrostatic screening between polycation and polyanion and the subsequent aggregation of formed PEC, which is described in an equivalent reaction by

$$
\begin{gathered}
\mathrm{PLL}^{-\mathrm{H}^{+}}+\mathrm{PLG}^{-} \rightleftharpoons \mathrm{PEC} \\
n \mathrm{PEC} \rightleftharpoons(\mathrm{PEC})_{n}
\end{gathered}
$$

Taking into consideration the dependenceof reciprocal chemical relaxation times on decreasing concentrations of polyelectrolytes leads to the origin (as shown in Figure 3), the backward reaction, written in eq 3 and 4 looks negligibly small. The slow decrease in LSI in the elapsed time over LSI $_{\max }$, observed at higher concentrations above 0.5 mequiv $^{-1}$ (Figure 1), may result from further rearrangement and growth of primary aggregates. In addition, the half reaction time between polycations and a luminescence-labeled protein, charged negatively, was $8.7 \mathrm{~s}$ in the presence of $6 \mathrm{mM} \mathrm{NaCl} .^{2}$ This relatively slow reaction, interesting to biological systems, seems to involve not only electrostatic shielding but other effcts, although concentrations of ionized groups and the mixing ratio of polycations and polyanions was unknown.

In summary, the PEC formation, worked on the change of light scattering intensity, was concluded to involve processes for pairing between polycations and polyanions and for their subsequent aggregation of the formed PEC.

Acknowledgements. The authors appreciate cordially Prof. Wolfram Schnabel, HahnMeitner Institut für Kernforschung Berlin $\mathrm{GmbH}$, for stimulative discussions and finan- 
cial support, and Dr. Tsuneo Okubo, Kyoto University, for progressive suggestion and discussion for this study.

\section{REFERENCES}

1. T. Okubo, K. Hongyo, and A. Enokida, J. Chem. Soc., Faraday Trans. 1, 80, 2087 (1984).

2. V. A. Izumrudov, A. Z. Zezin, and V. A. Kabanov,
Dokl. Akad. Nauk. SSSR, 291, 1127 (1986).

3. M. Eigen, Discuss. Faraday Soc., 17, 194 (1954).

4. A. Nakajima, K. Shinoda, T. Hayashi, and H. Sato, Polym. J., 7, 550 (1975).

5. A. Domard and M. Rinaudo, Macromolecules, 13, 898 (1980).

6. G. Beck, J. Kiwi, D. Lindenau, and W. Schnabel, Eur. Polym. J., 10, 1069 (1974).

7. H. Sato and A. Nakajima, Polym. J., 7, 241 (1975). 